\title{
Considerações acerca do uso de hidrogênio como gás de arraste para cromatografia gasosa
}

\author{
Carlos Henrique de Vasconcelos Fidelis \\ Departamento de Química Analítica, Instituto de Química, \\ Universidade Estadual de Campinas - UNICAMP \\ CP 6154, Cep 13083-970, Campinas, SP, Brasil \\ e-mail:chvf@yahoo.com
}

\section{Resumo}

A escolha do gás de arraste a ser utilizado em um cromatógrafo a gás deve considerar razões de ordem operacional, bem como de adequabilidade à aplicação pretendida, o que é função de propriedades físicoquímicas dos gases. Tanto hélio $(\mathrm{He})$ quanto hidrogênio $\left(\mathrm{H}_{2}\right)$ preenchem o requisito básico de um bom gás de arraste: ser um gás inerte. No entanto, os critérios de escolha vão além, sobretudo nos últimos anos, em que a baixa disponibilidade, devido à diminuição em sua produção, tem aumentado o custo do He. Esses fatores em conjunto têm motivado muitos pesquisadores a preferir hidrogênio a hélio como gás de arraste para utilização nos cromatógrafos a gás. Contudo, cada um desses gases apresenta características únicas e as implicações desta mudança devem ser entendidas e pesadas na hora da escolha. Uma série de dúvidas surge quando se considera a possibilidade da troca de nitrogênio ou hélio por hidrogênio. Os aspectos que envolvem eficiência, segurança, operacionalidade e custo são aqui discutidos.

Palavras-chave

Cromatografia gasosa; gás de arraste; hélio; hidrogênio.

\section{Considerations on the use of hydrogen as carrier gas for gas chromatography}

\section{Abstract}

The choice of carrier gas to be used in a gas chromatograph must consider operational reasons, as well as suitability for the intended application, which is a function of physical and chemical properties of gases. Both helium $(\mathrm{He})$ and hydrogen $\left(\mathrm{H}_{2}\right)$ meet the basic requirement of a good carrier gas: to be an inert gas. However, the selection criteria go further, especially in recent years, where the low availability, due to the decrease in its production, has increased the cost of He. These factors together have motivated many researchers to prefer hydrogen instead helium as carrier gas for use in gas chromatographs. However, each of these gases presents unique characteristics and implications of this change must be understood and weighed at the time of choice. A series of questions arises when one consider the possibility of exchange nitrogen or helium, by hydrogen. The issues involving efficiency, safety, operability and cost are discussed here.

Keywords

Gas chromatography; carrier gas; helium; hydrogen. 


\section{Introdução}

Hidrogênio, hélio e nitrogênio comportam-se quase idealmente nas pressões e temperaturas tipicamente utilizadas em cromatografia gasosa. Consequentemente, não influenciam a seletividade na cromatografia gás-líquido, exceto em condições extremas. A escolha do gás de arraste pode ainda afetar a resolução, entretanto por meio do seu efeito sobre a eficiência da coluna, que resulta de diferenças nas taxas de difusão de solutos. Pode ainda afetar tempos de retenção, porque velocidades ótimas de gás de arraste diminuem com o decréscimo da difusividade de solutos. O gás de arraste pode ter uma influência considerável na velocidade da análise cromatográfica, proporcional à difusividade molecular de um soluto no gás ${ }^{[1]}$. Tal influência depende da queda de pressão na coluna. Outras considerações a serem feitas na escolha de certo gás incluem custo, pureza, segurança, reatividade e compatibilidade com o detector.

Dentre os gases de arraste mais empregados em cromatografia gasosa, o nitrogênio poderia ser considerado a melhor escolha, considerando-se uma menor contribuição do termo $\mathrm{B}$ na equação de van Deemter (difusão molecular do soluto na fase móvel) quando comparado ao $\mathrm{H}_{2}$ e He. No entanto, esta eficiência estende-se sobre um intervalo estreito de velocidades lineares. Devem-se destacar ainda outras características do nitrogênio como sua viscosidade, que aumenta rapidamente com a temperatura, diminuindo a velocidade linear, tornando-se uma desvantagem principalmente quando se trata de corridas com programação de temperatura. Além disso, a difusividade de solutos, que influencia tanto a altura de prato quanto a velocidade ótima de gás de arraste descrita pela equação de van Deemter, é bem mais baixa em nitrogênio que em hélio e hidrogênio ${ }^{[2]}$. Essas observações são válidas para filmes finos de fases estacionárias $(<0,5 \mu \mathrm{m})$. As conclusões para maiores espessuras de fase estacionária são diferentes, uma vez que a difusão na fase estacionária contribui significativamente para o mecanismo de alargamento de picos. Nesse caso, fatores de retenção, temperatura e coeficiente de difusão na fase estacionária devem ser considerados na escolha do gás de arraste para uma separação em particular ${ }^{[2]}$.

Apesar de permitir a obtenção de menor altura de prato, a velocidade ótima do nitrogênio é muito baixa, levando a separações excessivamente lentas. Esses fatores em conjunto limitam o uso de nitrogênio como gás de arraste a situações muito particulares como, por exemplo, alguns métodos que empregam detector de captura de elétrons (ECD). Por outro lado, o hidrogênio mantém sua eficiência dentro de uma faixa bem mais ampla de velocidades lineares, adequando-se especialmente ao uso em cromatografia rápida e ultrarrápida ${ }^{[1,3]}$. Outra propriedade que o credencia à aplicação em separações onde se deseja reduzir os tempos de retenção são as altas velocidades lineares obtidas a baixas pressões, sem grande perda de resolução.

Devido a diferenças de viscosidade, um importante parâmetro cromatográfico que pode ser afetado pelo uso de um ou outro gás de arraste são os tempos de retenção. $\mathrm{O}$ hidrogênio possui cerca da metade da viscosidade de hélio ou nitrogênio na mesma temperatura (Figura 1). Dessa forma, o hidrogênio é de uso praticamente obrigatório para altas temperaturas de trabalho, e em colunas longas, de até $100 \mathrm{~m}$, como as usadas em análises de ésteres metílicos de ácidos graxos, ou em petroquímica. O hélio, por sua vez, apresenta valores intermediários entre os dois primeiros gases considerados, no que diz respeito a eficiência ${ }^{[4]}$. 


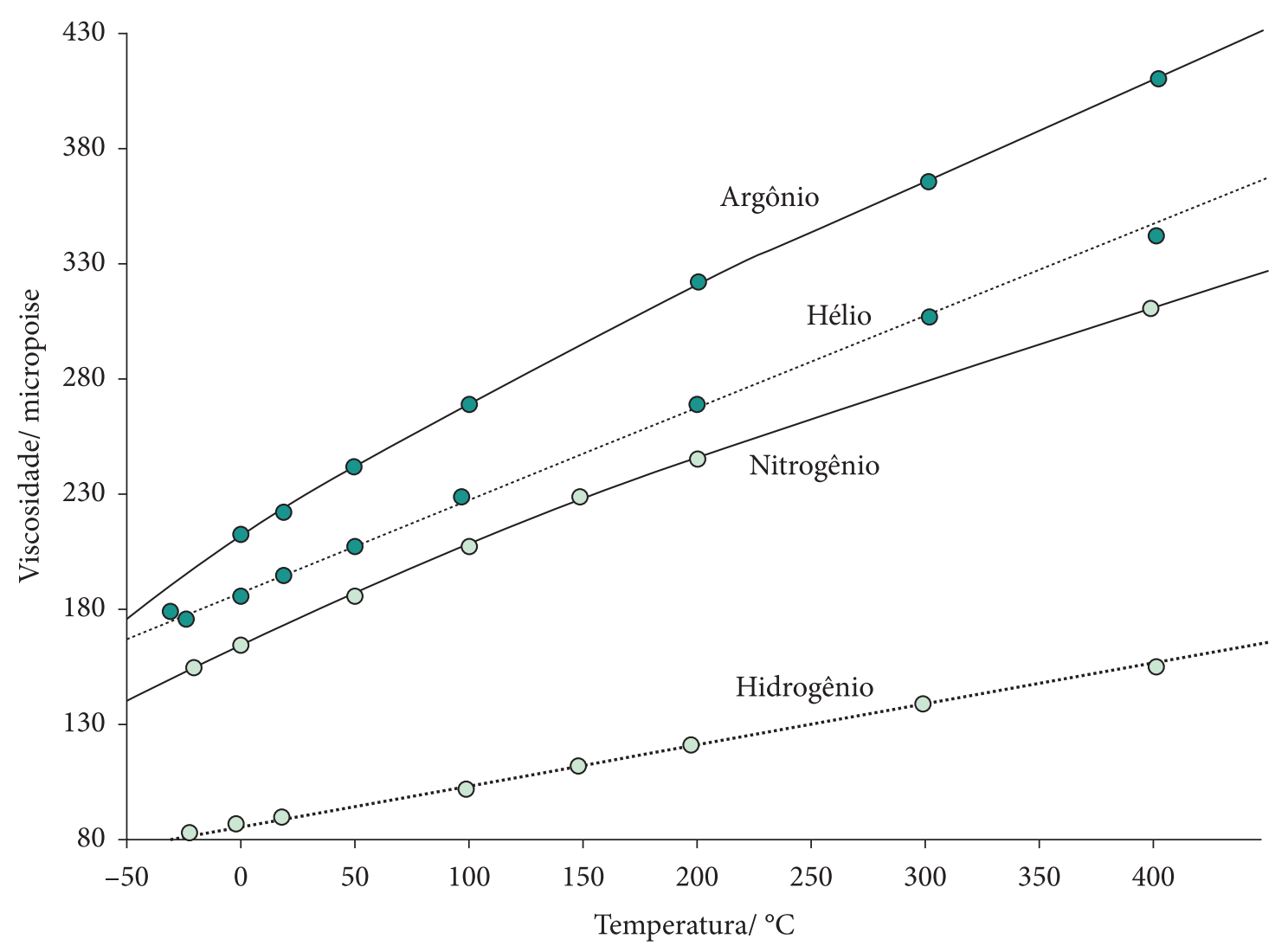

Figura 1 Efeito da temperatura na viscosidade de gases de arraste ${ }^{[5]}$.

Independentemente da questão das velocidades lineares, a maioria dos detectores são sensíveis a mudanças na vazão. O resultado mais evidente deste efeito são os deslocamentos na linha de base durante corridas com programação de temperatura. Para evitar tais inconvenientes, deve-se selecionar o gás com a menor razão viscosidade/coeficiente de difusão. Dentre os três gases considerados, também por este critério, o hidrogênio é a melhor escolha ${ }^{[5]}$.

Deve-se ainda considerar os aspectos que envolvem a segurança do hidrogênio para avaliar a extensão dos riscos envolvidos na sua utilização em cromatografia. Há soluções comerciais disponíveis para a utilização de hidrogênio de forma mais segura, como válvulas de segurança, sensores, geradores de hidrogênio com capacidade reduzida ${ }^{[6]}$, bem como procedimentos operacionais recomendados. O hidrogênio não pode ser usado com detectores termiônicos e em alguns espectrômetros de massas, mas a principal restrição ao seu uso refere-se à segurança, porque ele forma uma mistura explosiva com o ar. No entanto, algumas recomendações simples podem eliminar as restrições à sua utilização como gás de arraste, de tal maneira que é necessário ampliar o conhecimento a respeito dos fatores operacionais que envolvem o uso seguro de hidrogênio no laboratório. Em contrapartida, o custo do hélio tem sido um fator decisivo na escolha dos muitos pesquisadores que o têm preterido. O hélio, gás nobre e raro, não é produzido no Brasil, mas importado. Dentre os principais fornecedores encontram-se EUA, Argélia e Qatar ${ }^{[4]}$. Por outro lado, o hidrogênio, além de ser muito mais abundante, pode ser produzido pela eletrólise da água (juntamente com $\mathrm{O}_{2}$ ) em 
geradores de hidrogênio, pela catálise do metanol ou como subproduto de reações de desidrogenação.

O objetivo deste trabalho é apresentar e discutir as vantagens e desvantagens do uso de hidrogênio alternativamente ao hélio como gás de arraste em cromatografia gasosa, enfatizando seu emprego em cromatografia gasosa rápida e ultrarrápida.

\section{$2 \mathrm{He}$ vs. $\mathrm{H}_{2}$}

O maior receio quanto ao uso de hidrogênio como gás de arraste talvez seja quanto à segurança, uma vez que os químicos sabem da existência de reações explosivas em que o combustível é o hidrogênio. Comecemos então avaliando os aspectos relativos à segurança.

\section{1 Segurança}

Inicialmente é importante considerar que o hidrogênio requer o uso de tubos de aço na conexão do estoque de gás (geralmente um cilindro) com o cromatógrafo. Tubos de cobre sofrerão oxidação rapidamente, tornado-se frágeis.

Um mínimo de $4 \%$ de hidrogênio no ar é necessário para que ocorra uma explosão, à temperatura de cerca de $630{ }^{\circ} \mathrm{C}$. Nos equipamentos atuais, tais condições dificilmente estarão presentes, tornando o uso do hidrogênio bastante seguro. Ainda assim, são obrigatórias uma série de medidas que tornam seu emprego ainda mais seguro. Entre os maiores riscos encontram-se a quebra de colunas e os vazamentos, sobretudo aqueles em que o hidrogênio possa entrar em contato com partes aquecidas, como o injetor ${ }^{[7]}$. Dessa maneira, deve-se assegurar que não há vazamentos de qualquer natureza, antes de dar início às análises cromatográficas, bem como sempre fechar o cilindro que contém o hidrogênio quando o cromatógrafo não estiver em ope- ração. O uso de cilindros com capacidade reduzida é recomendável, como forma de minimizar o risco de acúmulo de hidrogênio em níveis críticos dentro do laboratório, em caso de vazamentos. Geradores de hidrogênio também evitam a estocagem de grandes quantidades sendo, no entanto, necessários investimentos relativamente altos em geradores que possam suprir a demanda do gás quando utilizado o modo split. Alguns outros aspectos que envolvem o uso seguro de hidrogênio são abordados a seguir.

\subsubsection{Vazão constante vs. pressão constante}

A regulagem do gás de arraste por vazão pode ajudar a prevenir acidentes, uma vez que o gás chegará ao forno (em caso de vazamento) em quantidade bastante limitada, principalmente quando comparada à situação em que o gás de arraste é controlado por pressão. Apesar do forno do cromatógrafo não ser hermeticamente fechado, um cálculo simples ajuda a estimar o tempo que levaria para uma mistura explosiva se formar em seu interior. Considerando um volume de $30 \mathrm{~L}$ para o forno de um cromatógrafo a gás convencional e uma vazão de $1 \mathrm{~mL} \mathrm{~min}^{-1}$, somente após cerca de 20 horas haveria $4 \%$ de hidrogênio dentro do forno. Por outro lado, vários litros do gás poderiam adentrar o forno em questão de poucas horas utilizado-se o controle por pressão. No entanto, voltando à realidade, felizmente o hidrogênio se difunde rapidamente e uma vez que o forno não é capaz de aprisionar considerável quantidade de gás em seu interior por tanto tempo, o risco de uma explosão nestas condições é praticamente nulo. Mesmo considerando um vazamento proveniente das altas vazões necessárias ao uso de altas razões de split, é improvável que a concentração de hidrogênio atinja níveis críticos dentro do forno nessas condições $^{[7]}$. 


\subsubsection{Sensores}

O uso de sensores não é ainda popular, porém traz uma segurança adicional. O funcionamento baseia-se na coleta de um pouco de ar do forno que é levado até um sensor que detecta hidrogênio em concentração de risco. Quando uma concentração de $1 \%$ é detectada, o cromatógrafo tem suas temperaturas baixadas, eliminando-se o risco de explosão ${ }^{[7]}$.

\subsubsection{Custo}

Enquanto o hidrogênio é bastante abundante e, apesar do custo elevado do alto consumo de energia elétrica, pode ser produzido com alto grau de pureza pela eletrólise da água, o hélio existe no ar em baixíssimas concentrações (cerca de 50 ppm). Além disso, os EUA, que produziam por volta de $90 \%$ do hélio consumido no mundo todo iniciaram, em meados da década de 90, um programa de privatização das reservas de hélio que resultou na diminuição da oferta desse gás. Concomitantemente houve um aumento na demanda nas últimas décadas, o que elevou ainda mais o preço do hélio. $O$ fator custo torna-se cada vez mais importante na escolha do gás de arraste, principalmente se o hélio puder ser substituído por um gás de menor custo sem perda na qualidade da análise cromatográfica. A viabilidade do uso de geradores de hidrogênio naturalmente depende da demanda do laboratório, mas a aquisição de um equipamento como esse pode se pagar em um período relativamente curto de tempo. Mesmo com o uso de cilindros de hidrogênio, a manutenção de um cromatógrafo a gás tende a reduzir a demanda dos valores necessários utilizando-se este gás ${ }^{[8]}$.

\subsection{Hidrogênio como gás de arraste}

A diferença na separação cromatográfica quando se compara hidrogênio e hélio não fica evidente ao confrontarmos as alturas equivalen- tes a um prato teórico (HETP), calculadas pela equação de van Deemter:

$\operatorname{HETP}=\mathrm{A}+\mathrm{B} / u+\mathrm{C} u$, onde:

- HETP é a altura equivalente a um prato teórico.

- $u$ = velocidade linear de gás de arraste ( $u=\mathrm{L} / \mathrm{t}_{\mathrm{M}}$; onde $\mathrm{L}$ é o comprimento da coluna e $t_{\mathrm{M}}$, o tempo de retenção de um composto não retido).

- A = constante que corresponde ao efeito de difusão das moléculas da amostra devido aos diferentes caminhos seguidos por elas (não utilizado em colunas capilares, considerando-se que há apenas um caminho a ser seguido).

- $\mathrm{B}=$ constante relacionada ao efeito de difusão molecular do soluto na fase móvel.

- $\mathrm{C}=$ constante proporcional à resistência da coluna à transferência de massa do soluto através da mesma.

A avaliação da constante B apontaria como preferível o uso de nitrogênio devido a sua maior densidade, que ocasiona uma diminuição na difusão do soluto e, assim, maior eficiência da coluna. A difusão excessiva é indesejável, pois causa alargamento dos picos.

O parâmetro expresso por essa equação, indicativo da eficiência (capacidade de separação) de uma coluna cromatográfica, possui valores mínimos semelhantes para ambos os gases (Figura 2).

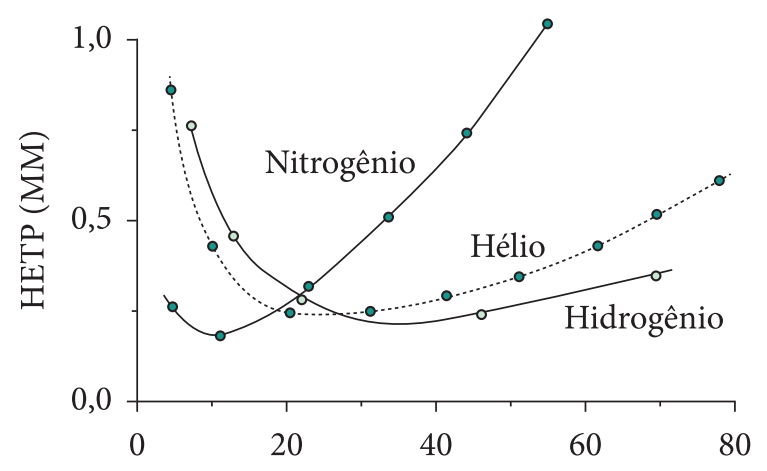

Figura 2 Gráfico HETP $\times$ velocidade linear média típica ${ }^{[5]}$. 
Menores valores indicam maior eficiência. Por outro lado, o hidrogênio pode ser usado em uma faixa mais ampla de velocidades lineares, sem grande perda de eficiência, o que pode ser usado para reduzir tempos de análise. A avaliação do uso de um ou de outro gás, no entanto, necessita de mais informações, tais como possíveis mudanças nos tempos de retenção.

\subsubsection{Tempos de retenção com hidrogênio e hélio}

Devido às diferenças nas propriedades físico-químicas apresentadas pelos dois gases, a ocorrência ou não de diferenças no tempo de retenção, quando comparadas corridas cromatográficas que difiram apenas quanto ao gás de arraste utilizado, deve ser estudada considerando velocidade, vazão constante e pressão de entrada constante, para então avaliar o que ocorre quando é utilizada programação de temperatura. A Tabela 1 resume as três situações. Mantendo-se constantes as dimensões da coluna capilar pode-se avaliar o porquê dos menores tempos de retenção obtidos com $\mathrm{H}_{2}$.

Para a operação isotérmica e velocidade linear média constante, tempos de retenção não são afetados pela mudança do gás de arraste, uma vez que o coeficiente de distribuição de um soluto na coluna não é afetado pelo tipo de gás utilizado.

A pressão pode ser limitante para a cromatografia gasosa, devido a fatores como a pressão mínima e máxima de operação de um cromató- grafo. Devido à baixa viscosidade, o hidrogênio requer menores pressões que hélio, considerando um mesmo diâmetro interno (D.I.) de coluna e há um ganho de tempo real de 60\% em relação ao hélio em condições de alta queda de pressão ${ }^{[1,3]}$. Em pressão constante, o uso de hidrogênio fará com que os picos eluam em cerca da metade do tempo, e, à vazão constante, os tempos de retenção de hidrogênio serão cerca de $78 \%$ dos obtidos usando hélio.

Em corridas com programação de temperatura, o efeito do uso de hélio ou hidrogênio nos tempos de retenção é diferente do observado na separação isotérmica, devido à mudança da viscosidade dos gases com a temperatura. Os efeitos nesse caso estão resumidos na Tabela 2.

Em pressão constante, deve-se observar que a eluição em menores temperaturas mudará a retenção relativa, causando mudanças em relação, por exemplo, ao cálculo de índices de retenção. Isso se deve à possibilidade de mudança de tempos de retenção relativos de compostos com características químicas diferentes, como diferentes polarida$\operatorname{des}^{[8]}$. À semelhança do efeito observado à vazão constante, o que ocorre à pressão constante deve-se a diferenças nas características físico-químicas, sobretudo a viscosidade, entre os dois gases. A diferença nas compressibilidades resulta em menores tempos de eluição dos analitos quando utilizado o hidrogênio e também em menores temperaturas.

$O$ hidrogênio oferece maior velocidade linear, permitindo menores tempos de retenção quando comparado ao hélio. A viscosidade dos

Tabela 1 Comparação do uso de $\mathrm{H}_{2}$ e He, controlando-se velocidade, pressão e vazão, em uma corrida isotérmica.

\begin{tabular}{ccc}
\hline Velocidade constante & Pressão constante & Vazão constante \\
\hline Mesmos tempos de retenção & $\mathrm{H}_{2}$ : Eluição em menores tempos de & Efeito intermediário entre \\
(isoterma). & retenção para alguns compostos & velocidade e pressão constantes: \\
Pressão de entrada para $\mathrm{H}_{2}=1 / 2$ & (aproximadamente $1 / 2$ do tempo & velocidade linear média com \\
pressão entrada necessária com He. & obtido com $\mathrm{He}$ ). & $\mathrm{H}_{2}=0,78$ velocidade com He. \\
\hline
\end{tabular}


Tabela 2 Comparação do uso de $\mathrm{H}_{2}$ e He, controlando-se velocidade, pressão e vazão, em uma corrida com programação de temperatura.

\begin{tabular}{ccc}
\hline $\begin{array}{l}\text { Velocidade constante } \\
\text { (mantida por sistema } \\
\text { pneumático eletrônico }\end{array}$ & Pressão constante & Vazão constante \\
\hline Mesmos tempos de retenção. & $\begin{array}{c}\mathrm{H}_{2} \text { : Elvição em menores tempos de } \\
\text { retenção para alguns compostos, } \\
\text { porém em menores temperaturas. }\end{array}$ & $\begin{array}{c}\text { Efeito semelhante ao observado } \\
\text { à pressão constante. }\end{array}$ \\
\hline
\end{tabular}

gases aumenta com a temperatura, causando uma diminuição na velocidade linear à pressão constante. A taxa de mudança na viscosidade com a temperatura é aproximadamente a mesma para os três gases. A viscosidade do gás determina a queda de pressão para uma dada velocidade ${ }^{[2]}$. Como $\mathrm{H}_{2}$ é menos viscoso que He (cerca da metade do valor para uma mesma temperatura: Figura 1), ele requer uma pressão menor para atingir a mesma velocidade. Assim, comparados à mesma pressão, $\mathrm{H}_{2}$ atinge maior velocidade. Esta relação de tempos entre $\mathrm{H}_{2}$ e He se mantém em aproximadamente 1:2, apesar da relação não linear entre velocidade e queda de pressão, devido a compressibilidade dos gases de arraste.

Se a separação cromatográfica é realizada com programação de temperatura e o gás de arraste é trocado de hélio para hidrogênio, pode ser necessário certificar-se da identidade dos compostos. A maneira prática de fazer isso é aumentando a velocidade da rampa de temperatura de forma a obter temperaturas de eluição semelhantes às obtidas com o hélio ${ }^{[8]}$.

\subsubsection{Sensibilidade do detector por ionização em chama com $\mathrm{H}_{2}$ como gás de arraste}

Em relação à vazão de hidrogênio no detector por ionização em chama (FID), que poderá ser alterada com o uso desse gás como gás de arraste, deverá ser ajustada de maneira a manter o valor recomendado, mantendo-se inalterada a sensibilidade do detector. No entanto, a alteração da sensibilidade do FID somente ocorrerá em velocidades lineares médias acima de $50 \mathrm{~cm} / \mathrm{s}$ ou pelo uso de colunas de D.I. > $320 \mathrm{~mm}$, situações nas quais a vazão pode cair o suficiente para alterar a sensibilidade do detector ${ }^{[8]}$. Quanto à pureza, este se torna um aspecto crítico se levarmos em conta que mesmo quantidades traço de oxigênio ou água atacam a fase estacionária de colunas capilares, sobretudo a altas temperaturas. Desta maneira, recomenda-se o uso de hidrogênio ultra puro 99,9999\%.

\subsubsection{Hidrogênio como gás de arraste para GC-MS}

Apesar do uso crescente do hidrogênio como gás de arraste como uma boa estratégia para acelerar análises cromatográficas, algumas limitações podem restringir seu uso quando a cromatografia gasosa encontra-se combinada com a espectrometria de massas (MS). Alguns pesquisadores inclusive não o adotam como forma de melhorar o emprego de GC-MS em análises de rotina ${ }^{[9]}$. De fato há mais argumentos contra seu uso que a favor.

Diferentemente de outros sistemas de detecção para cromatografia gasosa, no espectrômetro de massas a saída da coluna capilar encontra-se sob vácuo, reduzindo a pressão na cabeça da coluna necessária para manter certa vazão. $\mathrm{O}$ hidrogênio, que possui menor viscosidade que o hélio, irá permitir maior expansão de volume do 
solvente, podendo resultar em picos com cauda ou alargamento de picos $^{[10]}$. Outro problema que pode ocorrer é uma dificuldade da bomba de vácuo em eliminar o excesso de gás, favorecendo sua reação com fragmentos de íons (além do risco de explosão, apesar de muito baixo, como já discutido). Por este motivo recomenda-se fortemente que o forno do cromatógrafo seja desligado, bem como a vazão de hidrogênio seja reduzida a zero quando o sistema estiver fora de uso. Entretanto, melhores resultados são obtidos com colunas de diâmetro interno reduzido (como é típico em cromatografia rápida e em $\mathrm{GC} \times \mathrm{GC}^{[11]}$ ), bem como com técnicas de injeção em que a amostra adentra a coluna mais lentamente que em injeções splitless. A fim de evitar possíveis danos, bem como redução na sensibilidade do espectrômetro de massas, recomenda-se o uso de hidrogênio 99,9999\% de pureza.

\section{Cromatografia rápida}

Embora pareça não haver um consenso quanto à definição de cromatografia rápida, e embora definições baseadas somente no tempo de análise possam perder aspectos como a resolução ${ }^{[1]}$, a cromatografia rápida é uma modalidade que demanda altas velocidades de gás de arraste em relação às usadas em cromatografia gasosa convencional.

O uso de colunas com um D.I. reduzido é a opção mais lógica para uma separação mais rápida sem alteração significativa na resolução. Esta miniaturização reflete-se em uma maior eficiência por comprimento (L), uma vez que será diminuido proporcionalmente o valor mínimo de altura de prato $(\mathrm{H})^{[9,10]}$. Portanto, o comprimento da coluna pode ser diminuído pelo mesmo fator, a fim de produzir o mesmo número de prato.
Quando o D.I. é reduzido, a velocidade linear média ideal $(u)$ é maior, levando a um menor tempo de análise com a mesma capacidade de separação. No entanto, haverá uma capacidade de amostra muito menor e necessidade de maiores pressões de gás de arraste para realizar uma corrida, e a velocidade linear ficará limitada pela pressão que o equipamento pode atingir. Desta forma, em cromatografia rápida o hidrogênio é uma opção particularmente vantajosa, porque atinge maiores velocidades lineares que o hélio com menor perda de eficiência, e ainda em menores pressões ${ }^{[1]}$. Soma-se a esses fatores o coeficiente de difusão do hidrogênio, cujo valor resulta em análises mais rápidas em relação às que utilizam outros gases de arraste.

Cromatografia rápida envolve acentuadas quedas de pressão, principalmente quando o número de pratos teóricos necessários na separação pretendida é relativamente baixo ${ }^{[3]}$. Desconsiderando a tarefa de escolher a fase estacionária a ser utilizada, bem como da otimização das dimensões da coluna capilar, o hidrogênio assume um importante papel na viabilidade da cromatografia rápida. A Tabela 3 mostra a influência do tipo de gás de arraste em altos valores de queda de pressão. Os valores foram calculados usando coeficientes de difusão e viscosidades dinâmicas em equações descritas na literatura $^{[3]}$. Nota-se que o uso de hélio ou nitrogênio em altas velocidades as tornam mais distantes da velocidade linear ótima, em comparação ao hidrogênio.

Tabela 3 Tempo de análise para diferentes gases, em relação ao hidrogênio ${ }^{[3]}$.

\begin{tabular}{cc}
\hline Gás de arraste & Tempos relativos \\
\hline $\mathrm{H}_{2}$ & 1 \\
$\mathrm{He}$ & 1,6 \\
$\mathrm{~N}_{2}$ & 2,8 \\
\hline
\end{tabular}


Blumberg ${ }^{[12]}$ relatou cálculos semelhantes, que estimaram as vazões de velocidades ótimas relativas de hidrogênio e hélio, tendo sido apontada como $25 \%$ superior para o primeiro.

Em cromatografia rápida, as separações, além de muito rápidas, empregam rampas de temperatura com altas taxas de aquecimento. Isto torna o uso de $\mathrm{H}_{2}$ especialmente interessante, uma vez que o comportamento do gás de arraste em condições de programação de temperatura difere bastante do caso em que se utiliza uma isoterma, como discutido no item 2.2.1. Isso se deve à variação na viscosidade dos gases com a temperatura (Figura 1).

\section{Conclusões}

O hidrogênio pode ser a melhor escolha de gás de arraste em muitos casos, quando comparado ao hélio. A possibilidade de utilizar altas velocidades lineares sem grande perda de eficiência é a principal vantagem apresentada. Esse requisito é fundamental quando se trata de cromatografia gasosa rápida, na qual os valores de vazão, bem como as programações de temperatura com altas taxas de aquecimento, tornam o uso do hidrogênio praticamente mandatório. Desde que sejam observados alguns aspectos relativos a segurança, o uso do hidrogênio em cromatografia gasosa é bastante seguro. $\mathrm{O}$ emprego com detecção por espectrometria de massas, no entanto, é restrito devido ao interfaceamento com o espectrômetro, no qual a saída da coluna encontra-se sob vácuo. Pelas razões expostas e, ainda, pelo custo crescente do hélio, o hidrogênio tem se tornado o gás de arraste de preferência em um número cada vez maior de laboratórios.

\section{Referências}

1 Matisova E, Dömötörova, M. Fast gas chromatography and its use in trace analysis. Journal of Chromatography $A$
2003; 1000:199-221. http://dx.doi.org/10.1016/S00219673(03)00310-8

2 Poole CF. The essence of chromatography. Amsterdã: Elsevier; 2003. chapt. 2, p. 83-86.

3 Cramers CA, Leclercq PA. Strategies for speed optimisation in gas chromatography: an overview. Journal of Chromatography A 1999; 842; 3-1311. http://dx.doi.org/10.1016/S0021-9673(98)00894-2

4 Bartram RJ, Froehlich P. Considerations on Switching from Helium to Hydrogen. LCGC North America 2010; 28(10):890-900.

5 Grob RL, Barry, EF. Modern Practice of Gas Chromatography. 4rd ed. John Wiley \& Sons; 2004. chapt. 10. http://dx.doi.org/10.1002/0471651141

6 Parker Hannifin Corporation. In-House Generation of Hydrogen for Gas Chromatography 2009, LCGC. Online. Available from: http://chromatographyonline. findanalytichem.com/lcgc/article/articleDetail. jsp?id=581405

7 Grob K. Working Safely with Hydrogen as a Carrier Gas. Available from: http://www.restek.com/ Technical-Resources/Technical-Library/Editorial/ editorial_A016.

8 Hinshaw JV. Frequently Asked Questions about Hydrogen Carrier Gas. LCGC North America 2008. Available from: http://chromatographyonline. findanalytichem.com/lcgc/article/articleDetail. jsp?id=564647.

9 Maštovská K, Lehotay SJ. Practical approaches to fast gas chromatography-mass spectrometry. Journal of Chromatography A 2003; 1000:153-180. http://dx.doi. org/10.1016/S0021-9673(03)00448-5

10 Heseltine J. Hydrogen as a Carrier Gas for GC and GC-MS. LCGC North America 2010; 28:1. Available from: http://chromatographyonline.findanalytichem. com/lcgc/article/articleDetail.jsp?id=653133.

11 Pedroso MP, Godoy LAF, Fidelis CHV, Ferreira EC, Poppi, RJ, Augusto F. Cromatografia gasosa bidimensional abrangente (GC×GC). Quimica Nova 2009; 32:422-430. http://dx.doi.org/10.1590/ S0100-40422009000200029

12 Blumberg LM. Theory of fast capillary gas chromatography - Part 3: Column performance vs. gas flow rate. HRC-J. Journal of High Resolution Chromatography 1999; 22(7):403-413. http://dx.doi. org/10.1002/(SICI)1521-4168(19990701)22:7 